\title{
COMPARISON OF SUBMILLIMETER AND ULTRAVIOLET OBSERVATIONS OF NEUTRAL CARBON TOWARD ZETA OPHIUCHI
}

\author{
Jocelyn Keene, Geoffrey A. Blake, and T. G. Phillips \\ California Institute of Technology \\ Received 1986 April 28; accepted 1986 July 23
}

\begin{abstract}
We have observed the ${ }^{3} P_{1} \rightarrow{ }^{3} P_{0}$ ground state transition of $\mathrm{C}_{\mathrm{I}}$ emission toward $\zeta$ Oph. We compare this observation with predictions made from Copernicus ultraviolet absorption measurements of the population of the ${ }^{3} P_{1}$ level and with millimeter wave observations of $\mathrm{CO}$.

Subject headings: interstellar: matter — interstellar: molecules — stars: individual — ultraviolet: spectra
\end{abstract}

\section{INTRODUCTION}

We have observed the ${ }^{3} P_{1} \rightarrow{ }^{3} P_{0}$ line of neutral atomic carbon $(\mathrm{C}$ I) toward $\zeta$ Ophiuchi. This is the most sensitive observation made to date at the fine structure frequency of 492 $\mathrm{GHz}$. The $\mathrm{C}$ I emission line appears to be similar in spectral line shape to the ${ }^{12} \mathrm{CO}$ line observed toward $\zeta$ Oph by Liszt (1979).

This work has been carried out in order to relate submillimeter observations of the abundance of $\mathrm{C}_{\mathrm{I}}$ in interstellar clouds to ultraviolet observations. Because ultraviolet lines are observed in absorption against the spectra of bright background stars, the interstellar clouds that have been observed are of necessity diffuse and have little extinction. In contrast, submillimeter observations have typically been concentrated on dense clouds where emission lines are strong. The column densities of carbon measured by ultraviolet absorption lines (e.g., Jenkins and Shaya 1979; Jenkins, Jura, and Loewenstein 1983) have in most cases been less than $10^{16} \mathrm{~cm}^{-2}$, whereas the column densities measured by submillimeter emission lines (e.g., Phillips and Huggins 1981; Keene et al. 1985; Frerking et al. 1986) are almost all greater than $10^{17} \mathrm{~cm}^{-2}$. Until now there has been no overlap of sources.

It is important to relate the two types of measurements. For example, Tarafdar et al. (1985) predicted column densities of C I from their theoretical models of dynamically evolving clouds and compared the results to both ultraviolet and submillimeter observations of carbon abundance. For such uses it is necessary to establish that the two types of observations give the same results for a given region. Moreover, there are uncertainties associated with ultraviolet measurements of column densities. The curve of growth analysis of spectrally unresolved lines with large optical depths is particularly risky.

We chose to observe the $\zeta$ Oph diffuse cloud to initiate this project because there have been extensive studies of its ultraviolet C I lines (de Boer and Morton 1974, 1979). Also, in surveys of the $\mathrm{C}$ I abundance in many diffuse clouds (Jenkins and Shaya 1979; Jenkins, Jura, and Loewenstein 1983) the C I abundance toward $\zeta \mathrm{Oph}$ is one of the largest and best determined. The $\zeta$ Oph cloud has been extensively observed at many wavelengths and there are large compilations of its interstellar lines (e.g., Herbig 1968; Morton 1975). Comparisons of radio frequency molecular emission lines with ultraviolet or optical absorption-line studies of the same species - CO (Liszt 1979; Wannier, Penzias, and Jenkins 1982), CH (Lang and Willson 1978), and OH (Crutcher 1979)-indicate that the bulk of the diffuse cloud lies in front of the star, since, within the errors, the two techniques give the same answer for the column density of gas toward $\zeta$ Oph.

It is the aim of this paper to show that, for the $\zeta$ Oph cloud, the ultraviolet and submillimeter techniques measure similar C I column densities and line velocity, and to point out how high signal-to-noise ratio submillimeter observations in the future may aid in the interpretation of ultraviolet data.

\section{OBSERVATIONS}

Observations of the $\mathrm{C}$ I lines toward $\zeta$ Oph were made on the night of 1985 July 25 from NASA's Kuiper Airborne Observatory (KAO) at an altitude of $12.5 \mathrm{~km}$. We used the InSb cyclotron-resonance heterodyne receiver described by Brown, Keene, and Phillips (1985). A single channel with a bandwidth of $1 \mathrm{MHz}\left(0.6 \mathrm{~km} \mathrm{~s}^{-1}\right)$ was stepped along the spectrum by changing the frequency of the klystron local oscillator under computer control. The spacing between data samples was $0.5 \mathrm{~km} \mathrm{~s}^{-1}$.

We observed $\zeta$ Oph for $\sim 2.5 \mathrm{hr}$, which is as long as is practical for such a southern object on one flight of the KAO. We calibrated with observation of $290 \mathrm{~K}$ and $80 \mathrm{~K}$ loads to determine the receiver temperature $(350 \mathrm{~K})$ and with observations of the Moon and Jupiter to determine the beam and aperture efficiencies. We assumed the temperature of the subsolar point of the Moon to be $390 \mathrm{~K}$ and the submillimeter emissivity to be $97 \%$ (Linsky 1973) and measured the beam efficiency to be $48 \%$. All the values quoted for $T_{A}^{*}$ in this paper have been corrected for this beam efficiency. After the observations we mapped our beam, using Jupiter as a source, and found that we had been pointing at $\zeta$ Oph to within 0.25 of a beam width (actually $45^{\prime \prime}$ to the west). Since Jupiter is small compared to our beam diameter it can also be used to measure the beam profile and aperture efficiency. The measured beam FWHM was 3'. Assuming a temperature of $160 \mathrm{~K}$ for Jupiter at $609 \mu \mathrm{m}$ (Hildebrand et al. 1985) the aperture efficiency was $40 \%$. The system temperature on the sky (corrected for spillover and beam efficiency) was $880 \mathrm{~K}$. We estimate our absolute calibration uncertainty to be of the order of $25 \%$.

Usually, in the course of spectral-line observations, spatial switching is employed for the purpose of eliminating baseline variations due to changes in receiver gain or noise temperature across the bandpass. While beam switching does, for us, usually lead to good flat baselines, not far from zero, it also has the unfortunate effect of degrading the signal-to-noise ratio of 
the observations by a factor of 2 . Because we were able to calculate in advance, from ultraviolet observations of the population of the ${ }^{3} P_{1}$ level (de Boer and Morton 1974, 1979), the line temperature that could be expected, we realized that it would be difficult to detect the line if we employed the beamswitching method for these observations. Also, judging from examination of POSS plates, it looked as though the nearest good "off" position might be many degrees away. Beam switching such a large distance is impractical on the KAO, and undoubtedly would not have resulted in good baseline subtraction. The dominant form of baseline variation for our system is the systematic error known as standing waves. When these occur they are sinusoidal with a period of $44 \mathrm{MHz}\left(27 \mathrm{~km} \mathrm{~s}^{-1}\right.$ at $492 \mathrm{GHz}$ ). They are set up in the $3.4 \mathrm{~m}$ path length between the feedhorn and secondary mirror of the KAO and are due to a slight amount of klystron-local-oscillator noise power creeping into the receiver IF and being detected as signal. However, the klystron that we have been using recently for $\mathrm{C}$ I observations is of high quality and the amount of standing waves it introduces is very small. Also the velocity space that we scanned $\left(10 \mathrm{~km} \mathrm{~s}^{-1}\right)$ is less than half a standing wave period. Any standing wave present in the data would be varying smoothly and could not introduce spurious features on frequency scales of interest. For these reasons, we decided not to beam switch.

The rest frequency of the ${ }^{3} P_{1} \rightarrow{ }^{3} P_{0}$ transition of ${ }^{12} \mathrm{C}$ was determined initially by Saykally and Evenson (1980) to be $492.1623 \mathrm{GHz}$. Astronomical observations (Frerking et al. 1986) suggest that the frequency probably is somewhat lower, $\sim 492.1607 \mathrm{GHz}$. Recent new laboratory measurements and reanalysis of previous data have led to a revised laboratory frequency of $492.1612 \mathrm{GHz}$ (Cooksy et al. 1986), which agrees with the astronomical measurements to within the errors. In this paper we use the astronomically determined value, 492.1607 GHz.

\section{PREDICTIONS FROM ULTRAVIOLET MEASUREMENT OF C I}

We chose to observe $\zeta$ Oph in part because the column density of atomic carbon has been well established by de Boer and Morton $(1974,1979)$ who have made extensive studies of the C I ultraviolet lines as observed by Copernicus. They have determined the column density of $\mathrm{C}_{\mathrm{I}}$ in the three ground level states to be

$$
\begin{gathered}
{ }^{3} P_{0}, \ldots, 2 \times 10^{15} \mathrm{~cm}^{-2}, \quad{ }^{3} P_{1}, \ldots, 1 \times 10^{15} \mathrm{~cm}^{-2}, \\
{ }^{3} P_{2}, \ldots, 2 \times 10^{14} \mathrm{~cm}^{-2} .
\end{gathered}
$$

The quoted uncertainty in the ${ }^{3} P_{1}$ column density measurement is $25 \%$. However, a reevaluation of the data by de Boer (1979) showed that the populations of the upper two levels may have been underestimated by as much as $40 \%$ (see also $\S$ VI).

From the measured column density can be calculated the expected integrated intensity for emission in the ${ }^{3} P_{1} \rightarrow{ }^{3} P_{0}$ transition. The intensity, $I$, that is emitted in the transition is related to the column density in the ${ }^{3} P_{1}$ state, $N_{1}$, by

$$
\int I d v=\frac{h v}{4 \pi} N_{1} A_{10},
$$

but also

$$
\int T_{A}^{*} d V=\frac{c^{3}}{2 k v^{3}} \int I d v
$$

so that

$$
\int T_{A}^{*} d V=\frac{h c^{3}}{8 \pi k v^{2}} N_{1} A_{10}
$$

From the observed value of $N_{1}, 10^{15} \mathrm{~cm}^{-2}$, and the calculated value of $A_{10}, 7.9 \times 10^{-8} \mathrm{~s}^{-1}$ (Nussbaumer 1971; Nussbaumer and Rusca 1979), we find that the expected ${ }^{3} P_{1} \rightarrow{ }^{3} P_{0}$ line intensity is $0.17 \mathrm{~K} \mathrm{~km} \mathrm{~s}^{-1}$.

\section{RESULTS}

The $\mathrm{C}$ I spectrum shown in Figure 1 (heavy line) has had a quadratic baseline removed. The individual spectral points have a frequency resolution of $1 \mathrm{MHz}\left(0.6 \mathrm{~km} \mathrm{~s}^{-1}\right)$ and are spaced by $0.5 \mathrm{~km} \mathrm{~s}^{-1}$. The observed line is centered at $V_{\mathrm{LSR}} \approx$ $-0.5 \mathrm{~km} \mathrm{~s}^{-1}$.

The statistical noise level can be calculated from the system noise temperature and the integration time; the calculated $1 \sigma$ noise level is $\sim 0.06 \mathrm{~K}$, consistent with the observed rms level in the spectral points outside the line. The peak line temperature is $\sim 0.23 \mathrm{~K}$ and appears to be significant compared to the noise. The integrated line intensity is $0.40 \mathrm{~K} \mathrm{~km} \mathrm{~s}^{-1}$. Unfortunately, there is an uncertainty in the position of the baseline which particularly affects the estimate of the integrated line intensity. Since we did not measure "off" positions the baseline is defined only by the outermost spectral points. The unexpectedly large apparent line width made the number of channels outside the line smaller than anticipated. The uncertainty in the integrated intensity may therefore be as much as a factor of 2 . The measured intensity corresponds to a column density in the ${ }^{3} P_{1}$ state of $2.4 \times 10^{15} \mathrm{~cm}^{-1}$.

We consider that the agreement between the expected $(0.17$ $\left.\mathrm{K} \mathrm{km} \mathrm{s}^{-1}\right)$ and measured $\left(0.40 \mathrm{~K} \mathrm{~km} \mathrm{~s}^{-1}\right)$ intensities is acceptable given the uncertainties involved in the comparison. Not only are there uncertainties due to the rather low signal-tonoise ratio and the poorly determined baseline of the submillimeter observations, but there are others as well. The submillimeter measurement was made with a $3^{\prime}$ beam, compared to the pencil beam of the ultraviolet measurements. The submillimeter measurement samples the entire line of sight, both in front of and behind the star, whereas the ultraviolet measurements, of course, only sample space in front of the star. In addition, there may be uncertainties in the oscillator strengths for the ultraviolet transitions. Optical depth effects which also modify the comparison are discussed in $\S \mathrm{VI}$.

\section{COMPARISON WITH OBSERVATIONS OF CO}

Figure 1 (light line) shows the $J=1 \rightarrow 0$ spectrum of ${ }^{12} \mathrm{CO}$ by Liszt (1979; $1^{\prime}$ beam). The C I and CO spectra are similar in that both appear to have a narrow spike of emission at $\sim V_{\mathrm{LSR}}=-0.5 \mathrm{~km} \mathrm{~s}^{-1}$. The determination of the velocity of the C I spike is limited by the frequency resolution of the observations and by the accuracy with which the frequency of the ${ }^{3} P_{1} \rightarrow{ }^{3} P_{0}$ transition is known, as well as the signal-tonoise ratio. The difference in velocity between the $\mathrm{C}$ I and $\mathrm{CO}$ spikes is not significant. Both spectra also appear to have a lower intensity broad plateau of emission. In the case of $\mathrm{CO}$, the plateau appears only redward of the narrow spike, in $\mathrm{C}_{\mathrm{I}}$ it appears on both sides of the spike. The level of the plateau in the $\mathrm{C}$ I line is only marginally above the noise level, so its shape and intensity are somewhat in doubt. 


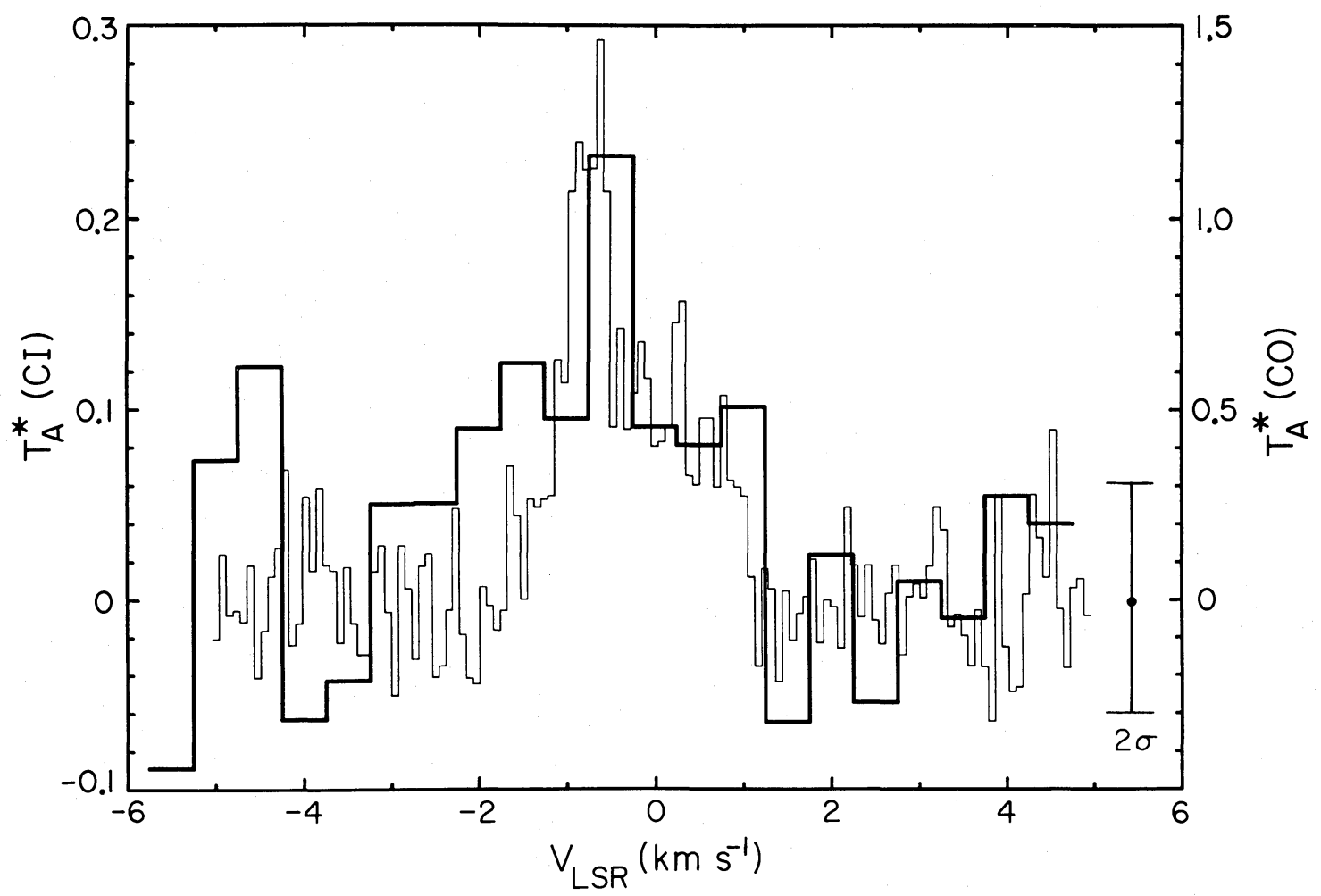

Fig. 1.-A comparison of the ${ }^{3} P_{1} \rightarrow{ }^{3} P_{0}$ spectrum of $C_{\mathrm{I}}$ (heavy line) observed toward $\zeta$ Oph with the $J=1 \rightarrow 0$ spectrum of CO (Liszt 1979 ; light line). The $\mathrm{C}$ I data points have a velocity resolution of $0.6 \mathrm{~km} \mathrm{~s}^{-1}$ but are spaced by $0.5 \mathrm{~km} \mathrm{~s}^{-1}$. The error bar on the right shows the $\pm 1 \sigma$ uncertainty for the $\mathrm{C}$ I measurements.

\section{COMPARISONS WITH ULTRAVIOLET MEASUREMENTS}

In the direction toward $\zeta$ Oph the difference between local standard of rest velocities and heliocentric velocities is $14.0 \mathrm{~km}$ $\mathrm{s}^{-1}$. The spike in the $\mathrm{C}$ I emission thus occurs at a heliocentric velocity $\left(V_{\text {hel }}\right)$ of about $-14.5 \mathrm{~km} \mathrm{~s}^{-1}$. The velocity structure of the clouds along the line of sight to $\zeta$ Oph has been analyzed by Hobbs (1973). He found clouds at heliocentric velocities of $-9.0,-12.6,-14.4$, and $-17.5 \mathrm{~km} \mathrm{~s}^{-1}$. Of these four clouds, the central two are the strongest, with the $-12.6 \mathrm{~km} \mathrm{~s}^{-1}$ being strong in the lines of $\mathrm{CH}^{+}$, and the $-14.4 \mathrm{~km} \mathrm{~s}^{-1}$ cloud being strong in the lines of $\mathrm{K} \mathrm{I}$ and $\mathrm{Ca}$ II. The analysis by Morton (1975) of ultraviolet lines observed with Copernicus showed that most neutral atoms and molecules are found in the -14.4 $\mathrm{km} \mathrm{s}^{-1}$ cloud. He suggested, in particular, that all the $C_{\text {I }}$ exists in this cloud. Our spectrum includes all of these velocities except $V_{\text {hel }}=-9.0 \mathrm{~km} \mathrm{~s}^{-1}$. In agreement with Morton's suggestion, $\mathrm{C}_{\mathrm{I}}$ is observed in only the $-14.4 \mathrm{~km} \mathrm{~s}^{-1}$ cloud.

Because of the high spectral resolution available with heterodyne measurements, submillimeter observations of $C_{\text {I can }}$ aid in the analysis of ultraviolet spectra. The resolution of ultraviolet spectrometers is not sufficient to resolve the spectral lines. Figure 1 shows details in the $V_{\text {hel }}=-14.4 \mathrm{~km} \mathrm{~s}^{-1}$ cloud that could not have been found in the ultraviolet spectra. At high resolution this cloud divides into two components: a narrow one with FWHM of $0.5 \mathrm{~km} \mathrm{~s}^{-1}$ or less $\left(b \leq 0.3 \mathrm{~km} \mathrm{~s}^{-1}\right)$ and a broader pedestal line with FWHM of $2-4 \mathrm{~km} \mathrm{~s}^{-1}$ $\left(b \approx 1.2-2.4 \mathrm{~km} \mathrm{~s}^{-1}\right)$.

The submillimeter C I emission is undoubtedly optically thin; the optical depth estimated using the level populations determined from ultraviolet lines and a line FWHM of $0.5 \mathrm{~km}$ $\mathrm{s}^{-1}$ is only 0.07 . In contrast, the ultraviolet $\mathrm{C} \mathrm{I}$ lines are not necessarily optically thin. In fact most of the ultraviolet $\mathrm{C}_{\mathrm{I}}$ lines lie on the flat portion of the curve of growth (de Boer and Morton 1974). In such a situation, as discussed by Nachman and Hobbs (1973), the curve of growth analysis of the ultraviolet lines is dominated by the higher velocity dispersion cloud; the effects of the narrow-line cloud may not be noticed at all. The most likely result is an underestimation of the total abundance of $\mathrm{C}$ I. This has been discussed previously by de Boer (1979) and Liszt $(1979,1981)$. In a reanalysis of earlier data, de Boer (1979) estimated that the carbon abundance for the excited fine-structure levels might be as much as $40 \%$ higher than that given by de Boer and Morton (1974 and 1979) if the $\mathrm{C}_{\mathrm{I}}$ lines are narrower than had been thought previously. In our observed spectrum the narrow emission spike contributes $\sim 20 \%$ of the total emission; in the higher signal-to-noise CO spectrum (Liszt 1979) it contributes at least $\sim 25 \%$. This implies that the true column density may be higher than that derived from ultraviolet observations by $\sim 25 \%-33 \%$, in agreement with the estimate by de Boer. Future high signal-tonoise ratio submillimeter observations of $\mathrm{C}_{\mathrm{I}}$ in diffuse clouds will undoubtedly aid in the interpretation of ultraviolet absorption lines.

\section{FUTURE WORK}

We believe that is very important to tie together two methods of determining the abundance of $\mathrm{C}_{\mathrm{I}}$ in the interstellar medium: ultraviolet absorption-line measurements and submillimeter emission-line measurements. The work presented here represents the best effort that at present can be made to observe the submillimeter emission from diffuse clouds. The only improvement possible with current technology would be 
to devote many flights of the KAO to this project. Unfortunately, observations of diffuse clouds will not be aided by the use of large ground-based submillimeter telescopes, several of which will go into operation this year, because such clouds are extensive and their antenna temperatures will not be increased by using a large telescope. Moreover, ground-based measurements will have the disadvantage of the poor transmission of the atmosphere. Large telescopes, however, are essential for narrowing the submillimeter beam and making the ultraviolet and submillimeter lines of sight more nearly comparable. Hopes for progress therefore must ride on the development of improved submillimeter receivers, and further in the future, a large submillimeter telescope either in an airplane or in space. When improved receivers are developed, submillimeter observations of $\mathrm{C}$ I will provide a useful supplement to ultraviolet measurements and will aid in their interpretation.

We are grateful to the crew and management of the NASA's KAO for the excellent flight without which it wouldn't have been possible to gather these data. We thank E. Brown and M. Wengler for their help in making these observations and $\mathrm{K}$. de Boer, E. Shaya, and E. van Dishoeck for their useful comments. Airborne astronomy at Caltech is supported by NASA under grant NAG 2-1.

\section{REFERENCES}

Brown, E. R., Keene, J., and Phillips, T. G. 1985, Internat. J. Infrared and Millimeter Waves, $\mathbf{6}, 1121$

Cooksy, A. L., Saykally, R. J., Brown, J. M., and Evenson, K. M. 1986, Ap. J., 305, L89.

Crutcher, R. M. 1979, Ap. J.(Letters), 231, L151.

de Boer, K. S. 1979, Ap. J., 229, 132.

de Boer, K. S., and Morton, D. C. 1974, Astr. Ap., 37, 305.

. 1979, Astr. Ap., 71, 141.

Frerking, M. A., Keene, J., Blake, G. A., Phillips, T. G., and Beichman, C. A. 1986, Ap. J., submitted.

Herbig, G. H. 1968, Zs. Ap., 68, 243.

Hildebrand, R. H., Loewenstein, R. F., Harper, D. A., Orton, G. S., Keene, J., and Whitcomb, S. E. 1985, Icarus, 64, 64.

Hobbs, L. M. 1973, Ap. J. (Letters), 180, L79.

Jenkins, E. B., Jura, M., and Loewenstein, M. 1983, Ap. J., 270, 88

Jenkins, E. B., and Shaya, E. J. 1979, Ap. J., 231, 55.
Keene, J., Blake, G. A., Phillips, T. G., Huggins, P. J., and Beichmann, C. A. 1985, Ap. J., 299, 967.

Lang, K. R., and Willson, R. F. 1978, Ap. J., 224, 125.

Linsky, J. L. 1973, Ap. J. Suppl., 25, 163

Liszt, H. S. 1979, Ap. J. (Letters), 233, L147.

. 1981, Ap. J.(Letters), 246, L147.

Morton, D. C. 1975 , Ap. J., 197, 85.

Nachman, P., and Hobbs, L. M. 1973, Ap. J., 182, 481.

Nussbaumer, H. 1971, Ap.J., 166, 411.

Nussbaumer, H., and Rusca, C. 1979, Astr. Ap., 72, 129

Phillips, T. G., and Huggins, P. J. 1981, Ap. J., 251, 533.

Saykally, R. J., and Evenson, K. M. 1980, Ap. J. (Letters), 238, L107.

Tarafdar, S. P., Prasad, S. S., Huntress, W. T., Villere, K. R., and Black, D. C. 1985, Ap. J., 289, 220.

Wannier, P. G., Penzias, A. A., and Jenkins, E. B. 1982, Ap. J., 254, 100

Geoffrey A. Blake: Space Science Laboratory, University of California, Berkeley, CA 94720

Jocelyn KeENe and T. G. PhILlips: California Institute of Technology, 320-47, Pasadena, CA 91125 